\title{
INCIDÊNCIA DOS ENCAMINHAMENTOS DE FISIOTERAPIA DAS UBS DE GURUPI - TO
}

\section{INCIDENCE OF PHYSIOTHERAPY REFERRALS AT UBS IN GURUPI - TO}

Leivia Lima de Morais ${ }^{1}$, Lucas França Marra², Rafaela de Carvalho Alves ${ }^{3}$, Marllos Peres de Melo ${ }^{4}$, Walmirton Bezerra D'Alessandro ${ }^{5}$, Eliara Firmina Maria Silva Alves ${ }^{6}$

\section{RESUMO}

As Unidades Básicas de Saúde (UBS) são todos os estabelecimentos de saúde que prestam ações de Atenção Básica $(A B)$ no âmbito do SUS, objetivando atender até $80 \%$ das necessidades de saúde da população, reduzindo encaminhamentos para hospitais de referência. $A$ fisioterapia na $A B$ atua na reabilitação de enfermidades a partir do encaminhamento médico, além da prevenção de agravos e promoção à saúde. O presente estudo objetivou verificar o quantitativo de encaminhamentos fisioterapêuticos realizados pelas UBS do município de Gurupi-TO no ano de 2019, na tentativa de identificar a demanda, bem como a disponibilidade de profissional para a celeridade no atendimento. Os dados foram coletados nas dependências da central de regulação da secretaria municipal de saúde após aprovação do relatório quadrimestral pelo Conselho Municipal de Saúde. Contabilizou-se 771 encaminhamentos advindos de 13 UBS, para o atendimento dos 7 profissionais fisioterapeutas lotados na secretaria municipal de saúde. Apesar da ausência de dados sobre o quantitativo de pacientes atendidos, pode-se concluir que a falta de profissionais ainda é entrave para a celeridade dos atendimentos, onde muitas vezes não há disponibilidade necessária para suprir a demanda dos encaminhamentos. Neste sentido, nota-se a delonga nos agendamentos dos encaminhamentos diante das listas de espera de cada UBS.

Palavras-chave: Sistema único de Saúde. Unidade Básica de Saúde. Núcleo Ampliado de Saúde da Família - Atenção Primária. Fisioterapia.

\section{ABSTRACT}

Basic Health Units (BHU) are all health establishments that provide primary care (PC) actions within the scope of Health Unic System (HUS), aiming to meet up to $80 \%$ of the population's health needs, reducing referrals to referral hospitals. Physical therapy in PC acts in the rehabilitation of illnesses from medical referral, in addition to the prevention of injuries and health promotion. The present study aims to verify the number of physiotherapeutic referrals made by BHU in the municipality of Gurupi-TO in 2019, in an attempt to identify the demand, as well as the availability of professionals for the speed of care. Data were collected on the premises of the regulation center of the municipal health department after approval of the quarterly report by the Municipal Health Council. 771 referrals were made from $13 \mathrm{BHU}$, for the assistance of the 7 physiotherapists working in the municipal health department. Despite the lack of data on the number of patients seen, it can be concluded that the lack of professionals is still an obstacle to the speed of care, where there is often no availability to meet the demand for referrals. In this sense, there is a delay in the scheduling of referrals before the waiting lists of each BHU.

Keywords: Health Unic System (HUS). Basic Health Unit (BHU). Expanded Family Health Center (EFHC-PC). Physiotherapy.
1 Fisioterapeuta. Residente no Programa de Residência Multiprofissional Integrada em Saúde da Família e Comunidade (Universidade de Gurupi - UnirG).

E-mail:

leiviamorais@hotmail.com

${ }^{2}$ Fisioterapeuta . Especialista em Saúde da Família Comunidade e Coletiva (IEP). Docente e Preceptor do Curso de Fisioterapia (UnirG).

${ }^{3}$ Mestre em Ciência da Saúde (UFT). Docente do curso de Fisioterapia (UnirG).

Doutor em Agronomia (UFT). Docente de Bioestatística da Universidade de Gurupi.

${ }^{5}$ Doutor em medicina tropical.

${ }^{6}$ Fisioterapeuta. 


\section{INTRODUÇÄO}

Com a implantação do Sistema Único de Saúde (SUS) em 1990, buscou-se melhorias no que diz respeito aos problemas de saúde da população brasileira, através de políticas públicas de saúde, programas e estratégias de aperfeiçoamento dos profissionais de saúde. Dentre as ações criadas, as Equipes de Saúde da Família (ESF) inseridas nas Unidades Básicas de Saúde (UBS), estabelecimentos de saúde, formam uma imensa rede de capilares os quais levam serviços de saúde no âmbito da atenção básica, objetivando atender até $80 \%$ das necessidades de saúde da população, muitas vezes não sendo necessário o encaminhamento para a média e alta complexidade. Desta forma a Atenção Básica ( $A B)$ e em especial a Estratégia Saúde da Família (ESF), para sua consecução, necessitam de diretrizes que apoiem as diferentes atividades a elas relacionadas, buscando promover a qualidade de vida da população brasileira e intervir nos fatores que colocam a saúde em risco, como falta de atividade física, má alimentação e o uso de tabaco, proporcionando atenção integral, equânime e contínua fortalecendo como uma porta de entrada do SUS. Ademais, o território adstrito coloca-se como alvo central para reorganizar o processo de trabalho em saúde mediante operações intersetoriais e ações de promoção, prevenção e atenção à saúde. ${ }^{1}$ Uma ferramenta para o auxílio no processo de trabalho das equipes de saúde foi, conforme a Política Nacional de Atenção Básica (PNAB), a criação dos Núcleos de Apoio à Saúde da Família (NASF) os quais são equipes multiprofissionais, podendo incluir profissionais tais como: médico acupunturista, assistente social, profissional/professor de educação física, farmacêutico, fisioterapeuta, fonoaudiólogo, médico ginecologista/obstetra, médico homeopata, nutricionista, médico pediatra, psicólogo, médico psiquiatra, terapeuta ocupacional, médico geriatra, médico internista (clínica médica), médico do trabalho, médico veterinário, profissional com formação em arte e educação (arte educador) e profissional de saúde sanitarista. Estes, devem atuar de maneira integrada e em apoio aos profissionais das equipes de Saúde da Família e às equipes de Atenção Básica, compartilhando práticas e saberes em saúde com essas equipes de referência apoiadas, buscando auxiliá-las no manejo ou resolução de problemas clínicos e sanitários, bem como agregando práticas, que ampliem o seu escopo de ofertas. ${ }^{2,3}$

O Núcleo Ampliado de Saúde da Família e Atenção Primária (NASF-AP) pode vincular-se à ESF e EAB (Equipe da Atenção Básica). Ele contribui para o aumento da 
capacidade do cuidado dessas equipes, compartilha problemas e maneja situações de saúde. Propõe-se a compartilhar ações através do apoio matricial por meio da dimensão técnico-pedagógica, cooperativista e horizontal que favorecerá a autonomia das equipes. Dessa forma, os profissionais das EAB podem desenvolver atividades com os profissionais do NASF e posteriormente sozinhos. Por exemplo, conduzir grupo de orientação postural, ensinar técnicas de respiração, lidar com usuário portador de sofrimento psíquico, realizar orientações nutricionais mais adequadas, orientar manobras de movimentação de usuário acamado, entre outras. ${ }^{1}$

Inicialmente a Fisioterapia direcionava-se para as práticas curativas e reabilitadoras, porém seu campo de atuação vem crescendo e os profissionais buscam aprimorar-se na prevenção de agravos e na promoção da saúde. A demanda por profissionais fisioterapeutas na atenção básica à saúde se faz cada vez mais necessária com o crescente aumento da população idosa, com suas patologias crônicas, e jovens susceptíveis a causas externas que os levam a lesões músculos-esqueléticas como acidente de trabalho, acidentes automobilísticos e envolvimento com a criminalidade. As doenças genéticas que acometem crianças e adolescentes também requerem cuidados fisioterapêuticos, tanto preventivos como mais específicos em reabilitação. ${ }^{5}$

A fisioterapia, como profissão da área da saúde, tem evoluído com mudanças no perfil de formação e atuação, ampliando o seu horizonte de ação, anteriormente reduzido ao caráter reabilitatório para uma visão mais integrativa do ser humano. $O$ fisioterapeuta detém importante papel no campo da reabilitação física, pois intensifica a recuperação física, contribui a melhora da funcionalidade, analgesia, em ações preventivas e proporciona uma melhor qualidade de vida. Porém, para que o fisioterapeuta consiga desempenhar esse papel corretamente, é necessário que conheça as condições epidemiológicas da população na área de abrangência, pois a epidemiologia busca compreender o processo saúde/doença. A fisioterapia na AB visa avaliar a ocorrência de certos tipos de enfermidades originadas nas atividades laborais e de vida diária atuando conjuntamente à ESF na prevenção de agravos e promoção à saúde, ou seja, quando se tem conhecimentos dos dados epidemiológicos, há mais possibilidades de desenvolver ações assertivas, bem como, o incentivo ao fisioterapeuta para que amplie suas ações além do âmbito curativo. ${ }^{6}$

Por isso, o levantamento da distribuição de encaminhamentos à fisioterapia das UBS do município de Gurupi - TO permite identificar a demanda pelo serviço, a 
proporcionalidade de encaminhamento em relação à quantidade populacional de cada região, assim como a disponibilidade de profissional para a celeridade no atendimento.

O objetivo desta pesquisa foi verificar o quantitativo de encaminhamentos fisioterapêuticos realizados pelas UBS no ano de 2019, na tentativa de identificar a demanda em determinados territórios de saúde adstrita de cada UBS em Gurupi Tocantins.

\section{MATERIAIS E METODOS}

Trata-se de uma pesquisa documental transversal realizada em Gurupi (Latitude: $11^{\circ} 43^{\prime} 30^{\prime \prime}$ Sul, Longitude: 49 4' 34" Oeste), município criado em 14 de novembro de 1958 pela Lei Estadual № 2.140, tendo como nome de origem Tupi Guarani que significa Diamante Puro, situada no sul do estado do Tocantins e de acordo com IBGE (2010) possui 76.755 habitantes (Figura 1) e estimativa de 86.647 habitantes para 2019. ${ }^{7}$

Após aprovação do relatório quadrimestral pelo Conselho Municipal de Saúde de Gurupi, os dados foram coletados nas dependências da central de regulação da secretaria de saúde do município e foram contabilizados o quantitativo de encaminhamento fisioterapêutico do ano de 2019 autorizado pelo médico regulador de acordo com cada UBS.

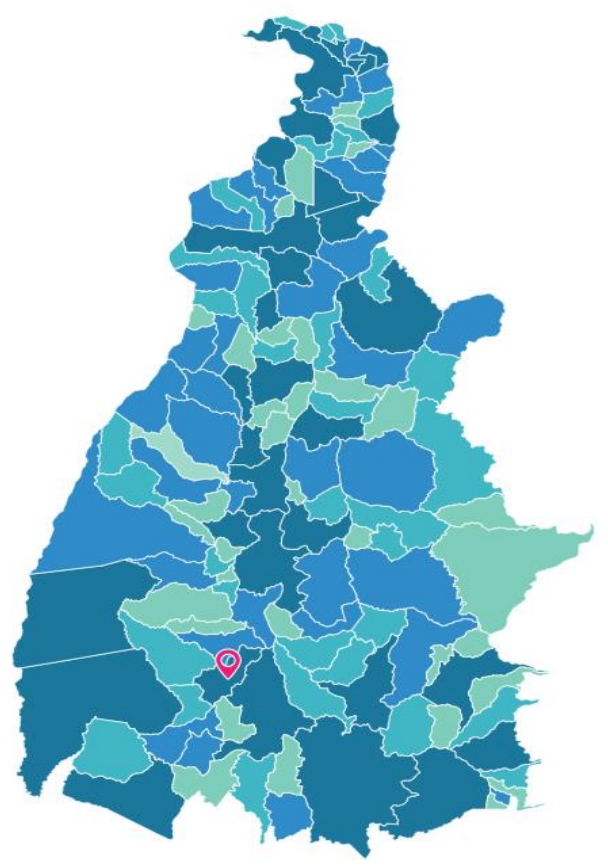

Figura 1- Município de Gurupi ao sul do Estado do Tocantins. Fonte: IBGE, 2010. Acessado em <https://cidades.ibge.gov.br/brasil/to/gurupi/panorama> Data: 30/06/2020. 
O município possui atualmente 15 UBS, porém no recorte da pesquisa, eram 13 unidades, as quais foram contabilizadas, sendo que 7 UBS é composta por profissionais fisioterapeutas membros da equipe do Núcleo Ampliado de Saúde da Família e Atenção Primária - NASF-AP (Figura 2). A figura ainda ilustra a divisão das UBS em 6 unidades do lado leste e 9 do lado oeste em relação a BR 153 que corta a cidade no sentido NORTESUL, sendo um total de 85.847 habitantes para 27-equipes de estratégia saúde da família - ESF e 1 Programa de Agentes Comunitários de Saúde - PACS

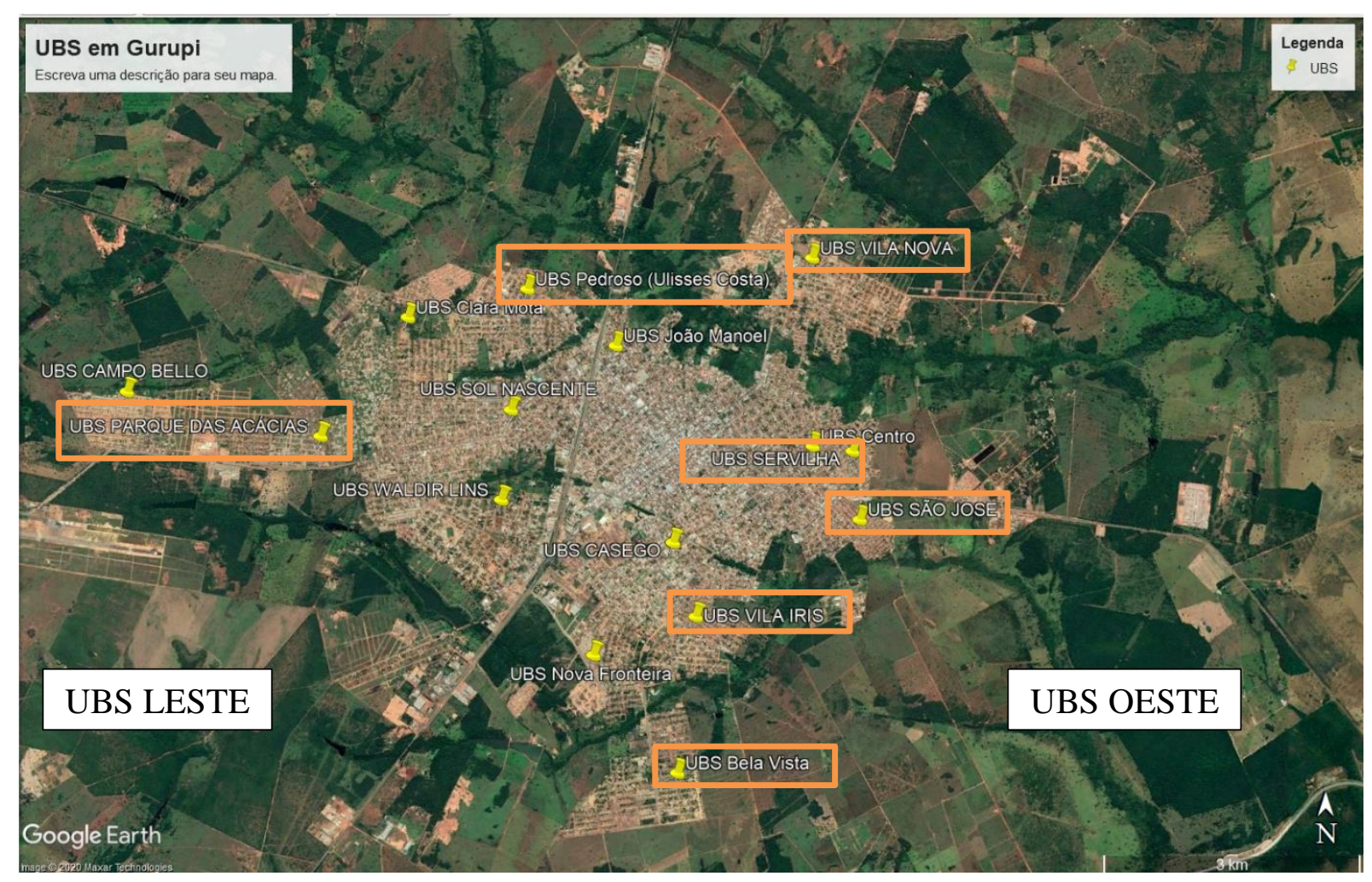

Figura 2- UBS localizadas no município de Gurupi e assinaladas devido à lotação do profissional fisioterapeuta. Fonte: Google Earth, 2020.

Foram incluídos encaminhamentos autorizados pelo médico regulador da secretaria municipal de saúde, emitidos pelos médicos da ESF das UBS de Gurupi -TO no período de janeiro a dezembro de 2019, período escolhido por conveniência, atualizado e aprovado pelos órgãos competentes até a coleta de dados para esta pesquisa. Foram excluídos encaminhamentos fora do período da coleta de dados e encaminhamentos não autorizados pelo médico regulador da secretaria municipal de saúde.

Por se tratar de dados secundários e públicos não houve necessidade de submissão ao Comitê de Ética em Pesquisa. Foram coletados dados à partir da 
publicação das Resoluções no 077/2019, no 020/2019 e no 005/2020 aprovadas pelo Conselho Municipal de Saúde de Gurupi - TO, referente ao ano de 2019.8

Os dados coletados foram tabulados em planilha Microsoft Excel® (2010), para análise descritiva em frequência, média, desvio padrão e percentual com elaboração de tabelas e gráficos.

\section{RESULTADOS}

O levantamento contabilizou setecentos e setenta e um (771) encaminhamentos fisioterapêuticos no ano de 2019 advindos de treze (13) unidades básicas de saúde, para o atendimento dos 7 profissionais fisioterapeutas lotados na secretaria municipal de saúde de Gurupi - TO.

A análise dos dados permitiu identificar que a quantidade de encaminhamentos não são proporcionais à quantidade de pacientes cadastrados nas UBS, ou seja, as particularidades da população de cada UBS, assim como a rotina de prescrição de cada profissional médico, determinam a demanda de encaminhamentos. Ocorreu proporcionalidade apenas na UBS Pedroso, que possui o maior quantitativo populacional e também de encaminhamentos.

A distribuição demográfica e geográfica entre leste e oeste, evidenciou que a região oeste possui maior quantitativo populacional, de encaminhamento e de profissionais fisioterapeutas. Comparando o quantitativo numérico entre população, encaminhamentos e profissionais, notou-se elevada discrepância entre a quantidade de populacional em relação à quantidade de profissionais fisioterapeutas, assim como há discrepância entre a quantidade de encaminhamentos em relação à quantidade de profissionais, ou seja, a região leste encontra-se com déficit profissional em relação à região oeste.

Para que haja proporcionalidade entre a quantidade de profissionais e o quantitativo de encaminhamentos, é necessário a contratação de mais um fisioterapeuta para a região leste.

Apesar da ausência de informações sobre a produtividade dos profissionais fisioterapeutas que atuam nas UBS, o relato dos mesmos permite identificar a escassez de profissionais, pois as listas de espera de pacientes para atendimento sempre são extensa. 


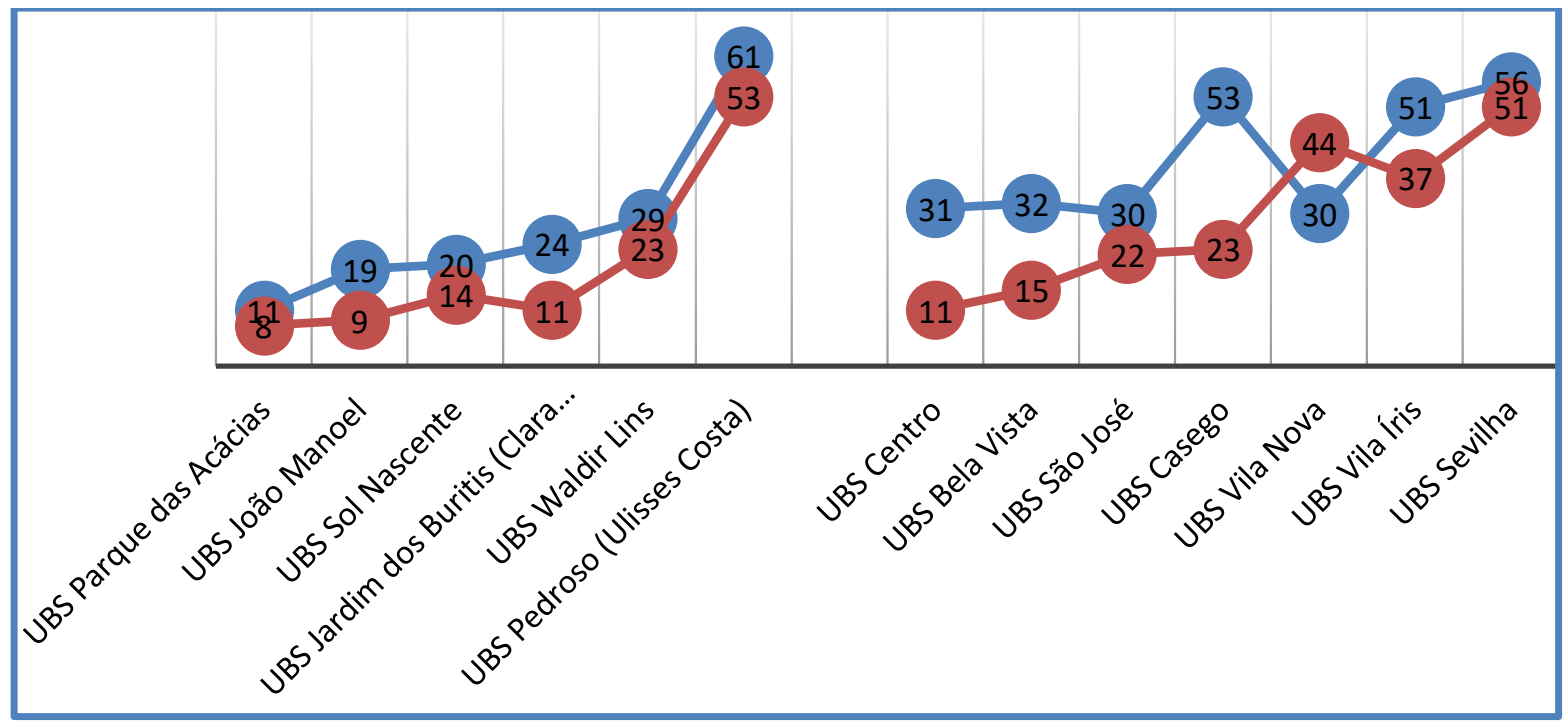

Figura 3- Quantitativo semestral de encaminhamentos à fisioterapia, distribuídos por UBS em Gurupi-TO.

Fonte: Elaboração própria.

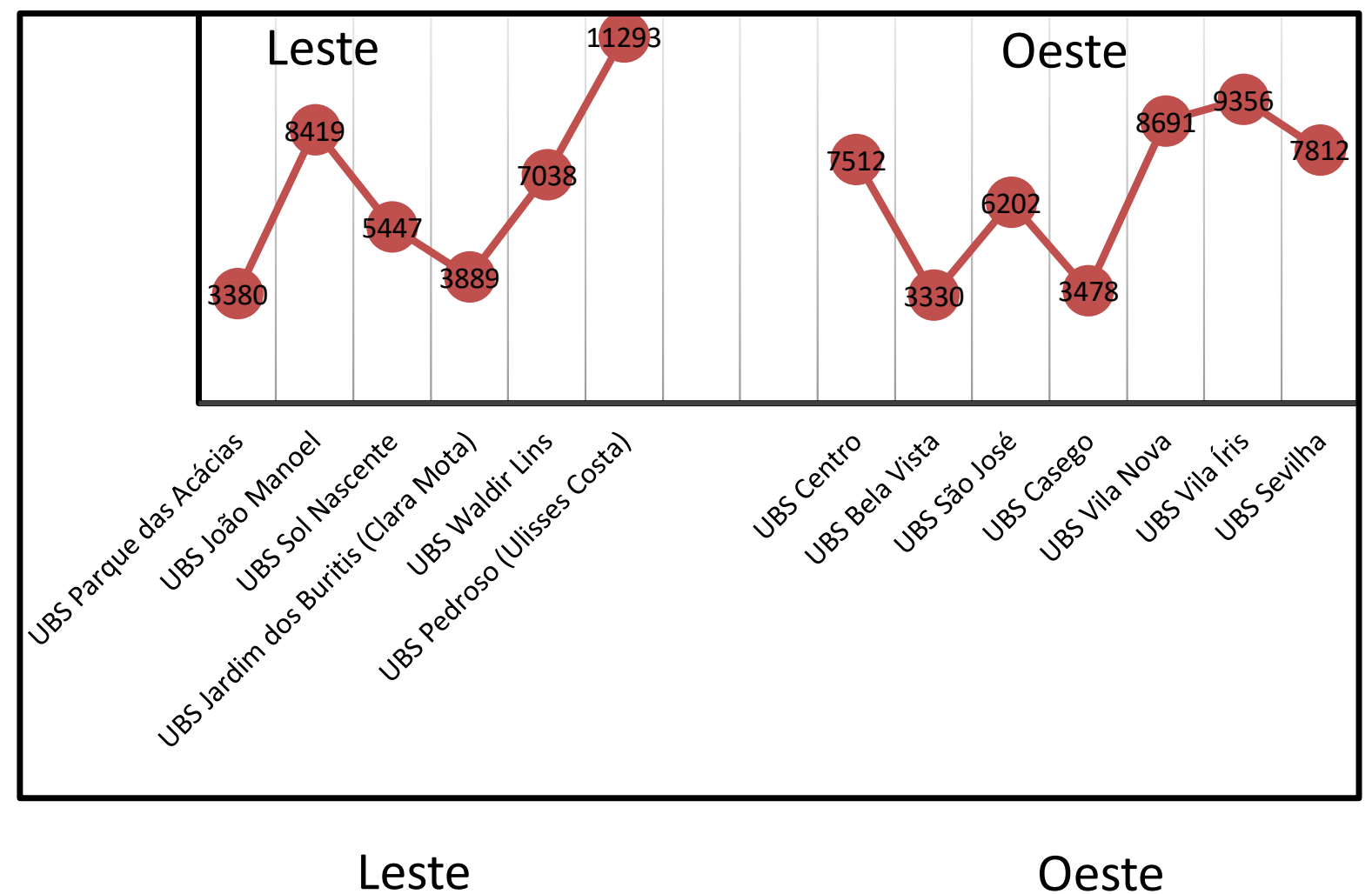

Figura 4- Número de pacientes cadastrados em cada UBS de Gurupi-TO.

Fonte: Elaboração própria.

\section{DISCUSSÃO}

A Política Nacional de Regulação está organizada em três dimensões integradas entre si: Regulação de Sistemas de Saúde, Regulação da Atenção à Saúde e Regulação do Acesso à Assistência, que devem ser desenvolvidas de forma dinâmica e 
integrada, com o objetivo de apoiar a organização do sistema de saúde brasileiro, otimizar os recursos disponíveis, qualificar a atenção e o acesso da população às ações e aos serviços de saúde. A Regulação do Acesso à Assistência, em especial, consiste na ordenação e qualificação dos fluxos de acesso às ações e serviços de saúde, de modo a otimizar a utilização dos recursos assistenciais disponíveis e promover a transparência, a integralidade e a equidade no acesso às ações e aos serviços, em tempo oportuno, dispondo, para tal, entre outros instrumentos, de diretrizes operacionais e protocolos de regulação. ${ }^{4}$

O profissional fisioterapeuta possui um importante papel no campo da reabilitação, visto que o conhecimento da biomecânica corporal contribui na melhora da funcionalidade dos pacientes, assim como a independência nas atividades de vida diária, proporcionando melhor qualidade de vida. ${ }^{9}$

Com relação ao atendimento fisioterapêutico, a Organização Mundial da Saúde (OMS) preconiza como ideal, a média de 1,5 mil habitantes por fisioterapeuta, porém, a média brasileira é de um fisioterapeuta para cada 8.500 habitantes. O Tocantins ainda está muito distante da realidade, todavia o número de fisioterapeutas atuantes vem aumentando nas diferentes Unidades de Saúde dos municípios, seja por meio de concursos ou por meio de contratos. ${ }^{10}$

Quanto à distribuição de profissionais fisioterapeutas e à demanda da população por esse profissional, faz-se necessário criar estratégias futuras voltadas para 0 desenvolvimento e para a redução das desigualdades regionais no que se refere à escassez de fisioterapeutas no território nacional. ${ }^{11}$

A importância do fisioterapeuta na APS influencia positivamente no atendimento das necessidades da população nos diversos ciclos de vida, colaborando para melhor resolutividade de problemas e promoção da saúde ainda neste nível de atenção, devido a soma de conhecimentos dentro das equipes, desenvolvendo sua autonomia olhando o indivíduo como um todo e não somente reabilitar a doença, mas desenvolvendo atividades tanto individual como em grupos, que favoreçam à prevenção de agravos. ${ }^{12}$

As ações desenvolvidas através das equipes multiprofissionais pressupõem a possibilidade da prática integrada, minimizando os impactos do processo saúde doença. A valorização dos diversos saberes e práticas contribui para uma abordagem mais integral e resolutiva para lidar com a dinâmica da vida social das famílias assistidas e da própria comunidade. Observou-se que nos municípios onde existe atuação do 
fisioterapeuta como importante profissional na composição da equipe de saúde da ESF ou NASF, uma contribuição significativa para a qualificação das ações de saúde junto à comunidade e a resolutividade de um sistema de saúde universal, integral e equitativo. ${ }^{13}$

Ademais o NASF-AB é essencial na manutenção da saúde visto que as demandas necessitam de diversas especialidades para aumentar a resolutividade e abrangência dos serviços ofertados pela $A B$ propondo visitas para acompanhamento do usuário, orientação aos cuidadores e às ACS no processo de reabilitação, visando a atenção com responsabilidade dos envolvidos no cuidado e não somente na dependência do usuário à presença do fisioterapeuta. O fisioterapeuta, segundo as diretrizes da ESF desenvolve ações promovendo serviços para tratamento e reabilitação, além da atuação na prevenção de agravos e doenças da população tanto de modo individual como em ações coletivas, por isso que é muito importante que aja uma boa estrutura física, materiais e capacitação dos profissionais isso influencia o andamento dos atendimentos favorecendo a um bom desfecho e levando a resolutividade das necessidades dos usuários do SUS. ${ }^{14,}$ 15,16

A ESF em conjunto com o NASF podem realizar discussões de casos clínicos entre $\mathrm{si}$, possibilitando atendimentos compartilhados como projetos terapêuticos ampliados e qualificados direcionados para as intervenções territoriais de grupos específicos realizados na própria UBS ou por meio de visitas domiciliares, na impossibilidade do usuário se deslocar até a UBS. Quanto à reabilitação, os profissionais do NASF com base no diagnóstico territorial, deverão apoiar as ESF com intuito de desenvolver ações de promoção e de proteção à saúde, subsidiando o acompanhamento destas ações voltadas para as deficiências populacionais. Para que estes objetivos sejam alcançados, o processo de trabalho dos profissionais do NASF descrito no caderno se dá por meio do apoio matricial, com a criação de espaços coletivos de discussão e planejamento. ${ }^{17}$

Ainda, as atuações do fisioterapeuta e do ACS com apoio da equipe multiprofissional ocorrem de forma interdisciplinar visando objetivos comuns como promoção de saúde e prevenção de doença, almejando a qualidade de vida da comunidade. Possuem atuações semelhantes como visitas domiciliares, ações educativas e orientações com abordagem individual e coletiva, realizadas nos domicílios e na comunidade como um todo. ${ }^{18}$

Aproximadamente $20 \%$ das consultas de médicos generalistas são relacionadas à distúrbios do sistema musculoesquelético, elevando a demanda por serviços de 
reabilitação em fisioterapia nas UBS, o que excede a capacidade dos serviços de reabilitação, ocasionando longo tempo de espera pelo tratamento e consequentes limitações no cuidado longitudinal e integral à saúde dos usuários. ${ }^{19}$

As ocorrências do usuário em relação ao acompanhamento dos serviços fisioterapêuticos ambulatoriais na saúde pública se dá frente às dificuldades de acesso, o longo tempo de espera pela assistência, as questões financeiras e burocráticas de encaminhamento e agendamento que interferem na continuidade do tratamento, a quantidade limitada de atendimentos e a dificuldade de deslocamento até o local, dando destaque também às dificuldades de locomoção física dos pacientes com deficiência motora como limitante para o acesso aos serviços públicos de reabilitação, face que os mesmos necessitam de terceiros para o transporte até a UBS. ${ }^{20}$

A expansão da $A B$ e o aumento dos estabelecimentos de saúde nos municípios podem ter impacto direto no crescimento e distribuição regional das profissões de nível superior, onde evidencia o fisioterapeuta como destaque. Um dos princípios da atenção básica é a descentralização e, assim, as equipes de Saúde da Família devem estar o mais perto possível dos usuários e dos serviços sendo a UBS o principal tipo de estabelecimento de saúde predominante nesse nível de atenção. ${ }^{21}$

\section{CONSIDERAÇÖES FINAIS}

Apesar da ausência de dados sobre o quantitativo de pacientes atendidos, pode-se concluir que a falta de profissionais ainda é um entrave para a celeridade dos atendimentos os quais, muitas vezes, não há disponibilidade necessária para suprir as demandas dos encaminhamentos. Neste sentido, nota-se a delonga nos agendamentos dos encaminhamentos diante das listas de espera de cada UBS.

Diante do contexto da ausência de informações sobre o quantitativo de pacientes atendidos, a Secretaria Municipal de Saúde (SEMUS) implementou a partir de janeiro de 2020 o sistema de registro de procedimentos, com objetivo de contabilizar a demanda suprida. Ainda com objetivo de resolutividade nos encaminhamentos fisioterapêuticos, a SEMUS adotou um novo fluxo de demanda, sendo que o direcionamento dos encaminhamentos da central de regulação está ocorrendo diretamente à central do NASF, tornando-se o coordenador do departamento de fisioterapia atento às demandas e agendamentos dos encaminhamentos destinados a cada UBS, minorando a fila de espera e a insatisfação dos usuários. 
Logo, faz-se necessário uma coleta de dados mais ampla e uma gestão mais eficiente buscando maior quantidade de profissionais para que seja otimizado os atendimentos, isso proporcionará melhor qualidade de vida para aqueles que necessitam do referido serviço.

\section{REFERENCIAS}

1. Ministério da Saúde (BRASIL). Portaria no 2436 de 21 de setembro de 2017. Aprova a Política Nacional de Atenção Básica, estabelecendo a revisão de diretrizes para a organização da Atenção Básica, no âmbito do Sistema Único de Saúde (SUS).

2. Langoni CS, Valmorbida LA, Resende TL. A introdução de atendimentos por fisioterapeutas em unidades da atenção primária em saúde. Rev. Bras. Promoção Saúde. v.25, n. 3, p. 261-270, 2012.

3. Ministério da Saúde (BRASIL). Política Nacional de Atenção Básica. 2011.

4. Ministério da Saúde (BRASIL). Portaria nº 1.559. Política Nacional de Regulação.2008.

5. Prado CS, Santos MR. Perfil dos pacientes atendidos no setor de fisioterapia do centro de atendimento secundário da prefeitura de Bragança Paulista. Trabalho de Conclusão de Curso, Bragança Paulista, 2017.

6. Oliveira AC, Braga DLC. Perfil epidemiológico dos pacientes atendidos na clínica de ortopedia da Universidade Paulista. Rev.J Health Sci Inst. Jun/Set, 2010.

7. IBGE. Censo Demográfico com estimativas populacionais para os municípios brasileiros, 2010. Disponível em: <www.ibge.gov.br>

8. Secretaria Municipal de Saúde (GURUPI). Prestação de Contas. 2020.

9. Dantas DRS, Silva M, Couto GS, Costa GS, Machado FLS, Junior FFUS, et al. Caracterização clínica dos pacientes com distúrbios musculoesqueléticos atendidos em um serviço público de reabilitação fisioterapêutica no município de São Francisco do Conde - Bahia. Rev. Ciênc. Méd. Biol., Salvador, v. 13, n. 2, p. 156-162, mai./ago. 2014.

10. Yamada AS, Noleto GR, Nunes RR, Cardoso FM, Adorno MLRG, et al. Levantamento de encaminhamentos médicos para fisioterapia em unidade básica de saúde do Tocantins. Revista Sítio Novo, Vol. 1, 2017.

11. Matsumura ESS, Júnior ASS, Guedes JA, Teixeira RC, Kietzer KS, Castro LSF. Distribuição territorial dos profissionais fisioterapeutas no Brasil. Fisioter Pesqui, 2018.

12. Batiston AP, Silva JS, Silva EC, Bonilha LAS, Ferrari FP, Fernandes JM, et al. Atuação do fisioterapeuta na atenção primária à saúde: o que sabem os agentes comunitários de saúde? Cad. Edu Saúde e Fis, 2019. 
13. Ribeiro CD, Soares MCF. Desafios para a inserção do fisioterapeuta na atenção básica: o olhar dos gestores. Revista de Salud Pública. Volumen 17 (3), Junio 2015.

14. Sales WB, Oliveira ASC, Pereira LEA, França JGM, Marcelino MC, Gerônimo CAS, et al. A importância da equipe NASF/AB enfretamentos e multidisciplinariedade: uma revisão narrativa/crítica. REAS/EJCH |Vol. Sup.n.48, 2020.

15. Freire LPV, Sales WB, Barbosa DS, Morais JD. As atribuições do fisioterapeuta do Núcleo Ampliado a Saúde da Família e Atenção Básica no município de Lucena-PB. Arch Health Invest 9, 2020.

16. Seus TL, Silveira DS, Tomasi E, Thumé E, Facchini LA, Siqueira FV. Estrutura para o trabalho e composição de equipes do Núcleo de Apoio à Saúde da Família: pesquisa nacional -Programa de Melhoria do Acesso e da Qualidade (PMAQ),2013. Epidemiol. Serv. Saude, Brasília, 2019.

17. Fernandes SCS, Ros MA. Desconstruir para transformar: o perfil do fisioterapeuta para o Núcleo de Apoio à Saúde da Família. Portal Editora Atlântica. Vol. 19, n. 2, 2018 http://dx.doi.org/10.33233/fb.v19i2.867

18. Aranha EAM, Oliveira VL, Rocha WA, Rizzo EP. fisioterapia na atenção básica da região metropolitana de vitória: inserção e desafios no psf. Revista Acadêmica Novo Milênio, v. 2, n. 1, p. 655-672, mar, 2020.

19. Meneses AS, Silva JSM, Silva LE. Perspectiva Financeira sobre Regulação de Filas de Espera para Fisioterapia na Atenção Primária à Saúde. SciELO Preprints, 2020.

20. Miranda RE, Schmidt D, Hanauer L, Peralles SRN, Striebell VL. Avaliação do acesso à fisioterapia após a alta hospitalar em indivíduos com Acidente Vascular Cerebral. Clin Biomed Res, 2018.

21. Carvalho MN, Gil CRR, Costa EMOD, Sakai MH, Leite SN. Necessidade e dinâmica da força de trabalho na Atenção Básica de Saúde no Brasil. Ciência \& Saúde Coletiva, 2018. 